\title{
TRATAMENTO TÉRMICO NO TEOR DE ANTOCIANINAS E CARACTERÍSTICAS SENSORIAIS DE BEBIDA NÃO ALCOÓLICA DE VINAGREIRA
}

\author{
Luma Moreira Martins, Lanamar de Almeida Carlos $^{2 *}$, Aline Arruda Gonçalves ${ }^{2}$, Kênia \\ Grasielle Oliveira $^{1} \&$ Caroline Liboreiro Paiva ${ }^{3}$
}

MARTINS, L. M.; CARLOS, L.A.; GONÇALVES, A.A.; OLIVEIRA, K.G. \& PAIVA, C.L. Tratamento térmico no teor de antocianinas e características sensoriais de bebida não alcoólica de vinagreira. Perspectivas Online: Biológicas \& Saúde. v. 8, n 27, p.27-37, 2018.

\section{RESUMO}

Este trabalho teve como objetivo avaliar a influência do tratamento térmico nas propriedades físico-químicas, sensoriais e no teor de antocianinas em uma bebida não-alcoólica elaborada com cálices de vinagreira (Hibiscus sabdariffa L.). Preparou-se uma formulação contendo $20 \%$ de cálices de vinagreira in natura, $15 \%$ de açúcar de cana (sacarose) e $65 \%$ de água mineral. As bebidas in natura e as provenientes dos tratamentos térmicos, pasteurização e esterilização foram avaliadas quanto as características físico-químicas (acidez total titulável, sólidos solúveis totais, $\mathrm{pH}$, sólidos totais), colorimétricas ( $\mathrm{L}^{*} \mathrm{e} \mathrm{a}^{*}$ ), sensoriais e quanto ao teor antocianinas totais. Não houve diferença significativa entre as bebidas termicamente processadas e a bebida in natura, em relação às características físico-químicas, exceto o $\mathrm{pH}$, que foi inferior para o produto esterilizado. A bebida esterilizada obteve as menores médias de aceitação em relação aos atributos cor, sabor, impressão global e também intenção de compra, configurando rejeição dos avaliadores. As antocianinas presentes na bebida pasteurizada foram significativamente preservadas e as da bebida esterilizada atingiram o nível de $89,81 \%$ de degradação. Conclui-se que a pasteurização é o tratamento térmico mais indicado para a bebida não-alcoólica de cálices de vinagreira, já que esta não diferiu da bebida in natura, em relação ao conteúdo de antocianinas e às características físico-químicas e sensoriais.

Palavras-chave: Hortaliça não convencional; Hibiscus sabdariffa L.; Processamento térmico; Propriedades sensoriais. 


\begin{abstract}
The aim of this work was to evaluate the influence of thermal treatment on the physical-chemical, sensorial properties and the anthocyanins content in a non-alcoholic beverage made with roselle (Hibiscus sabdariffa L.). A formulation was prepared containing $20 \%$ of in natura roselle calyces, $15 \%$ cane sugar (sucrose) and 65\% mineral water. Physico-chemical (total titratable acidity, total soluble solids, $\mathrm{pH}$, total solids), colorimetric ( $\mathrm{L}$ and $\mathrm{a} *$ ), and sensory properties and anthocyanins content of beveranges were investigated. The results of this study showed that there were no significant differences between the thermally processed beverages and the in natura beverage in relation to the physicochemical

lower for the sterilized product. The sterilized beverage obtained the lowest averages of the attributes color, taste and overall impression of acceptance, setting up rejection of the evaluators. The anthocyanins present in the pasteurized beverage were significantly preserved and those of the sterilized beverage reached the level of $89.81 \%$ degradation. It is concluded that pasteurization is the most suitable thermal treatment for the non-alcoholic beverage of roselle chalices, since it did not differ from the drink in natura, in relation to the anthocyanin content, the physical-chemical and sensorial characteristics, presenting higher averages acceptability.
\end{abstract} characteristics, except for the $\mathrm{pH}$, which was

Keyword: Unconventional vegetables; Hibiscus sabdariffa L.; Thermal treatments; Sensory properties.

1 Programa de Pós Graduação em Ciências Agrárias - Universidade Federal de São João Del-Rei - Campus Sete Lagoas - MG-424, Km 47, s/n - Indústrias, Sete Lagoas - MG, CEP: 35701-970- Brasil.

2 Departamento de Engenharia de Alimentos - Universidade Federal de São João Del-Rei - Campus Sete Lagoas - MG424, Km 47, s/n - Indústrias, Sete Lagoas - MG, CEP: 35701-970 - Brasil.

3 Instituto de Ciências Agrárias - Universidade Federal de Minas Gerais - Montes Claros - Av. Universitária, 1000 Montes Claros, MG, CEP: 39404-547 - Brasil.

(*)e-mail: lanamar@ufsj.edu.br

Data de chegada: 13/06/2018 Aceito para publicação: 20/09/2018 


\section{INTRODUÇÃ̃o}

A preocupação dos consumidores vem crescendo com relação às qualidades nutricionais e sensoriais dos produtos industrializados (GOBBI, 2016). Dentre esses produtos estão os sucos e as bebidas à base de vegetais, por apresentarem vitaminas, sais minerais e antioxidantes naturais. A aplicação de técnicas de processamento e de conservação podem interferir nas características originais dos produtos alimentícios, alterando suas características nutricionais e sensoriais (CIANCI et al., 2005), inclusive em bebidas à base de vegetais (ALVARENGA, 2017).

O consumo de néctares, em especial, vem crescendo à taxas significativamente maiores do que as de suco, provavelmente condicionado a confusão na distinção entre a diferença do produto (néctar/suco), custos ou preferência sensorial, aliada a informações insuficientes nos rótulos e que podem induzir ao aumento no consumo desses alimentos. Segundo dados da Associação Brasileira das Indústrias de Refrigerantes (ABIR), os sabores de bebidas não-alcoólicas e envasadas mais comercializados são uva, pêssego, laranja e maracujá (PIRILLO \& SABIO, 2009; LONGO-SILVA et al., 2015).

Dentre as técnicas de conservação mais utilizadas para a conservação de sucos, destaca-se a pasteurização, que tem por finalidade eliminar microrganismos patogênicos e alteradores, promovendo menores alterações nutricionais e sensoriais quando comparadas a métodos mais drásticos de processamento (JIAO, CASSANO, DRIOLI, 2004). Outro método de conservação por calor muito utilizado é a esterilização que se baseia na inativação de microrganismos patogênicos e deteriorantes que possam estar presentes no alimento, porém, envolve o emprego de altas temperaturas, o que pode levar a degradação de componentes presentes nas matrizes alimentícias, ainda que este aquecimento se dê por um curto período de tempo (VILLADIEGO et al., 2012).

A globalização e a industrialização vêm gerando mudanças nos hábitos alimentares do consumidor, que nem sempre são saudáveis e que podem estar relacionados ao surgimento de doenças (CARVALHO et al.,2006). Em contrapartida, o aumento da ingestão de produtos de origem vegetal diminui o risco de doenças crônicas não transmissíveis (DCNT), por conterem em sua estrutura, compostos que exercem atividade antioxidante com capacidade de neutralizar radicais livres (SILVA et al., 2010). Dessa forma, produtos alimentícios industrializados que contenham frutas e hortaliças em sua composição, como sucos, blends, chás gelados dentre outros, podem ocupar um lugar de destaque na preferência do consumidor.

As hortaliças não convencionais, também denominadas plantas comestíveis não convencionais (PANC), são de suma importância na alimentação humana por fornecerem minerais, vitaminas e fibras, porém, apresentam distribuição restrita a algumas localidades, fazendo parte da cultura e da alimentação de povos tradicionais (OLIVEIRA et al., 2013; KINUPP \& BARROS, 2008; MAPA, 2010; KINUPP \& LORENZI, 2014).

A vinagreira (Hibiscus spp) é considerada uma hortaliça não convencional, de origem africana, pertencente à família Malvaceae, a mesma do quiabo e do algodão (FONSECA et. al., 2016). O gênero Hibiscus, apresenta distribuição em áreas tropicais e subtropicais e encerra muitas espécies ornamentais, cuja principal atração é a flor. Dentre as estruturas vegetais do hibisco, a flor e o cálice são os de maior interesse. O cálice é um tecido carnoso, de sabor ácido e de coloração vermelha intensa, devido à presença de antocianinas. É bastante utilizado na elaboração de sucos, geleias, saladas, doces e outros tipos de produtos (NACHTIGALL \& ZAMBIAZI, 2006).

No Brasil, a vinagreira de folhas verdes (Hibiscus sabdariffa L.) é conhecida em várias regiões como vinagreira, grosela, caruru azedo, roselha, hibisco, hibiscus (MAPA, 2010), sendo que no Maranhão é utilizada como ingrediente no preparo de vários pratos típicos, como o cuxá e o arroz de vinagreira. No México é chamado como flor da Jamaica, na França roselle e na Nigéria, onde os cálices de hibisco são destinados ao preparo de bebidas, é conhecida como zobo (FONSECA, et al., 2016). 
O hibisco tem despertado grande interesse tanto da indústria farmacêutica como da alimentícia, por proporcionar benefícios à saúde, devido principalmente, a presença de compostos bioativos como vitaminas C e E, ácidos fenólicos e - flavonoides em sua constituição (WANG et al., 2000; VIZZOTTO \& PEREIRA, 2008). No grupo dos flavonoides, estão incluídas as antocianinas, que são responsáveis pela coloração dos vegetais e que apresentam elevada ação antioxidante, além da potencial utilização como corante pela indústria alimentícia (CARDOSO, LEITE, PELUZIO, 2011).

Um dos principais interesses da indústria alimentícia é aumentar a durabilidade de um produto pelo maior tempo possível, sem que ocorram alterações em suas qualidades sensoriais e nutricionais, para preservar ao máximo suas características originais. Nesse contexto, o uso de tratamentos térmicos, como a pasteurização ou a esterilização, destacam-se entre as alternativas que podem ser empregadas para esse fim. No entanto, há divergências a esse respeito, pois alguns estudos mostraram que o uso de calor, pode provocar alterações nas características sensoriais, físico-químicas e no teor de compostos bioativos de frutos, de hortaliças e de seus derivados (PALLET et al., 2005; MAIA et al., 2007; DAMASCENO et al., 2008; BRITO et al., 2011; ALVARENGA, 2017).

Sendo assim, objetivou-se, nessa pesquisa avaliar a influência do tratamento térmico (pasteurização e esterilização) sobre as características físico-químicas, sensoriais e o teor de antocianinas, em bebida nãoalcoólica, elaborada com cálices de vinagreira.

\section{MATERIAL E MÉTODOS}

\subsection{Material vegetal}

Os cálices de vinagreira (Hibiscus sabdariffa L.) utilizados para o processamento das bebidas foram colhidos no Banco de Hortaliças não Convencionais da Empresa de Pesquisa Agropecuária de Minas Gerais (EPAMIG), Fazenda Santa Rita, situada no município de Prudente de Morais-MG $\left(19^{\circ} 45^{\prime} 41.35^{\prime \prime} \mathrm{S}\right.$ $44^{\circ} 15^{\prime} 73.7^{\prime \prime}$ O). O material vegetal foi transportado sob refrigeração, para o Laboratório de Conservação de Alimentos, da Universidade Federal de São João del-Rei, Campus Sete Lagoas, onde foram devidamente preparados. Os cálices foram manualmente separados dos caroços, com o auxílio de facas de aço inoxidável, selecionados, lavados, sanitizados em solução aquosa de hipoclorito de sódio ( $200 \mathrm{ppm}$ de cloro ativo) e reservados.

\subsection{Elaboração da bebida-não-alcoólica de vinagreira}

Para a elaboração da bebida foram utilizados $20 \%$ de cálices de vinagreira in natura, $15 \%$ de açúcar de cana e água mineral em quantidade suficiente para se completar o volume final de 4 litros. Procedeu-se à homogeneização dos ingredientes em liquidificador industrial (METVISA) e em seguida, a bebida foi tamizada.

Apesar da bebida processada, ter apresentado $\mathrm{pH}<4$, optou-se por avaliar também o efeito da esterilização, visando obter informações acerca da aceitabilidade e do conteúdo de antocianinas de um produto, que pudesse apresentar vida de prateleira superior à do produto pasteurizado.

Uma parte da bebida in natura (sem tratamento térmico) foi utilizada para controle, outra parte foi submetida à pasteurização lenta, utilizando-se o binômio $80^{\circ} \mathrm{C}$, por 30 minutos e uma terceira parte, foi esterilizada em autoclave, utilizando-se o binômio $121^{\circ} \mathrm{C}$, por 15 minutos. As amostras para as análises físico-químicas foram retiradas, acondicionadas em tubos FALCON e armazenadas a $-40{ }^{\circ} \mathrm{C}$, em ultra freezer, até o momento das análises, que consistiu em um período máximo de 48 horas. As amostras destinadas à análise sensorial, foram resfriadas imediatamente após o processamento térmico, 
acondicionadas em garrafas esterilizadas e mantidas à $7{ }^{\circ} \mathrm{C} \pm 2$ em estufa tipo B.O.D., até o momento de serem servidas.

As bebidas foram refrigeradas a $7{ }^{\circ} \mathrm{C}$, em estufas tipo B.O.D. e, posteriormente, submetidas às avaliações físico-químicas, sensoriais e do conteúdo de antocianinas.

\subsection{Avaliação das características físico-químicas}

\subsubsection{Sólidos solúveis totais}

O teor de sólidos solúveis totais foi determinado por meio de refratometria, colocando-se gotas da amostra filtrada, sobre a lente de um refratômetro digital (REICHERT, R2 MINI), com compensação automática de temperatura (AOAC, 2012). Os resultados foram expressos em ${ }^{\circ} \mathrm{BRIX}$.

\subsubsection{Potencial hidrogeniônico}

$\mathrm{O}$ pH das amostras foi determinado por potenciometria, com o auxílio de um pHmetro digital (TEKNA, T-1000), por meio da imersão direta do eletrodo na amostra (AOAC, 2012).

\subsubsection{Acidez total titulável}

A acidez total titulável foi avaliada segundo a metodologia descrita pela AOAC (2012), que envolve a titulação da amostra com $\mathrm{NaOH} 0,1 \mathrm{~N}$, até viragem do indicador. Neste caso, a titulação foi assessorada pela medição do $\mathrm{pH}$ (até atingir o $\mathrm{pH}$ de 8,2), devido ao fato da cor da bebida ser a mesma formada pelo indicador fenolftaleína no ponto de viragem. Os resultados foram expressos em percentagem de ácido cítrico.

\subsubsection{Sólidos totais}

A determinação do conteúdo de sólidos totais foi realizada por gravimetria, baseando-se nas diferenças entre massas das amostras, antes e após serem submetidas à secagem em estufa à vácuo (TECNAL, TE-395), sob temperatura de $70{ }^{\circ} \mathrm{C}$, até massa constante (AOAC, 2012).

\subsubsection{Cor instrumental}

Realizou-se a análise dos parâmetros $\mathrm{L}^{*}$, $\mathrm{a}^{*} \mathrm{e} \mathrm{b}^{*}$, por meio de medição da coloração instrumental com o auxílio de um colorímetro (KONICA MINOLTA, CR410) E para tal, as amostras foram colocadas em um acessório de vidro apropriado para líquidos.

\subsubsection{Teor de antocianinas totais}

A quantificação de antocianinas totais, foi realizada pelo método espectrofotométrico do $\mathrm{pH}$ diferencial, conforme a metodologia descrita por Giusti \& Wrolstad (2001), com as leituras em comprimentos de ondas 510 e $700 \mathrm{~nm}$ em espectrofotômetro (FEMTO, 700S). Os resultados foram expressos em equivalência de cianidina-3-glicosídeo.

\subsection{Análise Sensorial}

Com intuito de avaliar a aceitabilidade e a intenção de compra das bebidas elaboradas, foi realizado um teste sensorial de aceitação e de intenção de compra, com 100 avaliadores não treinados, no Laboratório de Análise Sensorial, do Departamento de Engenharia de Alimentos, da Universidade Federal de São João del-Rei, Campus Sete Lagoas. 
No teste sensorial de aceitação foi utilizada a escala hedônica verbal estruturada de 9 pontos, com os extremos variando de "gostei extremamente" até "desgostei extremamente", para os atributos sabor, cor e impressão global. Para a avaliação da intenção de compra dos avaliadores, foi utilizada uma escala de 5 pontos, variando de "certamente não compraria" a "certamente compraria".

As amostras foram servidas de forma monádica, em copos descartáveis de $50 \mathrm{~mL}$, codificados com números de três dígitos e em temperatura de refrigeração. A avaliação foi feita em cabines individuais e sob luz branca (REIS e MINIM, 2013).

\subsection{Análise Estatística}

$\mathrm{O}$ experimento foi conduzido em delineamento inteiramente casualizado, com três repetições e em triplicata. Os valores obtidos foram submetidos à análise de variância (ANOVA) e as médias foram comparadas pelo teste de Tukey, ao nível de $5 \%$ de probabilidade.

\section{RESULTADOS E DISCUSSÃO}

\subsection{Características físico-químicas}

Os processamentos térmicos, que envolveram a pasteurização e a esterilização, não interferiram significativamente $(\mathrm{p}>0,05)$ na acidez total titulável, sólidos solúveis e sólidos totais, quando comparados entre si e com as amostras que não receberam tratamento térmico, conforme apresentado na Tabela 1. Resultados semelhantes foram relatados por Maia et al. (2007), para suco de acerola submetido à pasteurização. Já em estudos realizados com suco de laranja, observou-se ligeira diminuição de pH em sucos esterilizados quando comparados com a bebida in natura (FIORE et al., 2005), corroborando com os resultados observados nesse estudo, em que os valores de $\mathrm{pH}$ das bebidas esterilizadas e in natura apresentaram diferenças significativas (Tabela1). A natureza ácida dos cálices de hibisco, garantiu a obtenção de bebidas com pH inferior a 4,0 (Tabela 1), que é de grande importância para indústria de alimentos, uma vez que restringe o crescimento de microrganismos deteriorantes (VERA et al., 2003).

Tabela 1: Médias de acidez total titulável (ATT), de sólidos solúveis totais (SST), de pH e de sólidos totais (ST), de bebidas não-alcoólicas de cálices de vinagreira.

\begin{tabular}{ccccc}
\hline & \multicolumn{4}{c}{ Características físico-químicas } \\
\cline { 2 - 5 } Bebida & $\begin{array}{c}\text { ATT(\% de ácido } \\
\text { cítrico) }\end{array}$ & SST ( ${ }^{\circ}$ Brix $)$ & pH & ST $(\%)$ \\
\hline In natura & $0,13 \mathrm{a}$ & $11,76 \mathrm{a}$ & $2,65 \mathrm{a}$ & $11,6 \mathrm{a}$ \\
Pasteurizado & $0,16 \mathrm{a}$ & $13,43 \mathrm{a}$ & $2,58 \mathrm{ab}$ & $15,37 \mathrm{a}$ \\
Esterilizado & $0,13 \mathrm{a}$ & $12,8 \mathrm{a}$ & $2,51 \mathrm{~b}$ & $12,94 \mathrm{a}$ \\
\hline
\end{tabular}

*Médias seguidas de mesma letra na coluna não diferem entre si, ao nível de 5\% de significância pelo teste de Tukey.

Os parâmetros colorimétricos $\mathrm{L}^{*}$ \{que referem-se à luminosidade [variando de $-\mathrm{L}^{*}$ (preto) a $+\mathrm{L}^{*}$ (branco)] \} e $\mathrm{a}^{*}\left\{\right.$ que se refere ao croma $\mathrm{a}^{*}$ [variando de $-\mathrm{a}^{*}$ (verde) a $+\mathrm{a}^{*}$ (vermelho)], mostraram diferença significativa entre os tratamentos (Tabela 2). A bebida esterilizada apresentou maior luminosidade ( $\mathrm{L}^{*}$ ) quando comparada às bebidas pasteurizada e in natura, mas as colorações destas últimas não diferiram entre si (Tabela 2). As bebidas pasteurizadas e in natura, apresentaram maiores valores de a*, indicando maior 
intensidade da coloração vermelha quando comparada à bebida esterilizada, ou seja, houve uma diminuição da concentração dos pigmentos vermelhos (antocianinas) na bebida submetida ao processo de esterilização.

Tabela 2: Médias dos parâmetros colorimétricos $\mathrm{L}^{*}\left(-\mathrm{L}^{*}\right.$ preto ao $+\mathrm{L}^{*}$ branco) e $\mathrm{a}^{*}\left(-\mathrm{a}^{*}\right.$ verde ao $+\mathrm{a}^{*}$ vermelho), de bebidas não-alcoólicas de cálices de vinagreira.

\begin{tabular}{clc}
\hline & \multicolumn{2}{c}{ Parâmetros colorimétricos } \\
\hline Bebida & $\mathrm{L}^{*}$ & $\mathrm{a}^{*}$ \\
\cline { 2 - 3 } In natura & $20,01 \mathrm{~b}$ & $6,28 \mathrm{a}$ \\
Pasteurizada & $20,20 \mathrm{~b}$ & $5,85 \mathrm{a}$ \\
Esterilizada & $21,66 \mathrm{a}$ & $4,97 \mathrm{~b}$ \\
\hline
\end{tabular}

*Médias seguidas de mesma letra, na coluna, não diferem entre si, ao nível de 5\% de significância pelo teste de Tukey.

Estudos realizados por Damasceno et al. (2008), com sucos de maçã submetidos aos processos de pasteurização e de esterilização, mostraram que quando o suco foi submetido à temperatura de $121^{\circ} \mathrm{C}$ houve um escurecimento não enzimático, diminuindo assim, o valor de $\mathrm{L}^{*}$, efeito contrário ao observado para a bebida elaborada com cálices de vinagreira, desenvolvida nesse estudo.

As alterações dos parâmetros colorimétricos $\mathrm{L}^{*} \mathrm{e} \mathrm{a}^{*}$ apresentadas pelo produto esterilizado, refletem a degradação dos pigmentos naturais, oriundos da matriz alimentícia, ou seja, das antocianinas presentes nos cálices de vinagreira, conforme pode ser observado na Figura 1. Alterações dos pigmentos naturais, após aplicação de tratamento térmico, também foram observados em amostras de purê de frutas (BRITO et al. 2011) e em blends de capuchinha e maracujá (ALVARENGA, 2017).

\subsection{Teor de antocianinas totais}

A bebida esterilizada apresentou o menor teor de antocianinas $(0,31 \mathrm{mg}$ de cianidina-3-glicosídeo/L) em relação às demais, provavelmente devido à degradação promovida pela alta temperatura aplicada. A bebida in natura apresentou teor de antocianinas (3,04 mg de cianidina-3-glicosídeo/L) estatisticamente igual à pasteurizada (2,41 mg de cianidina-3-glicosídeo/L).

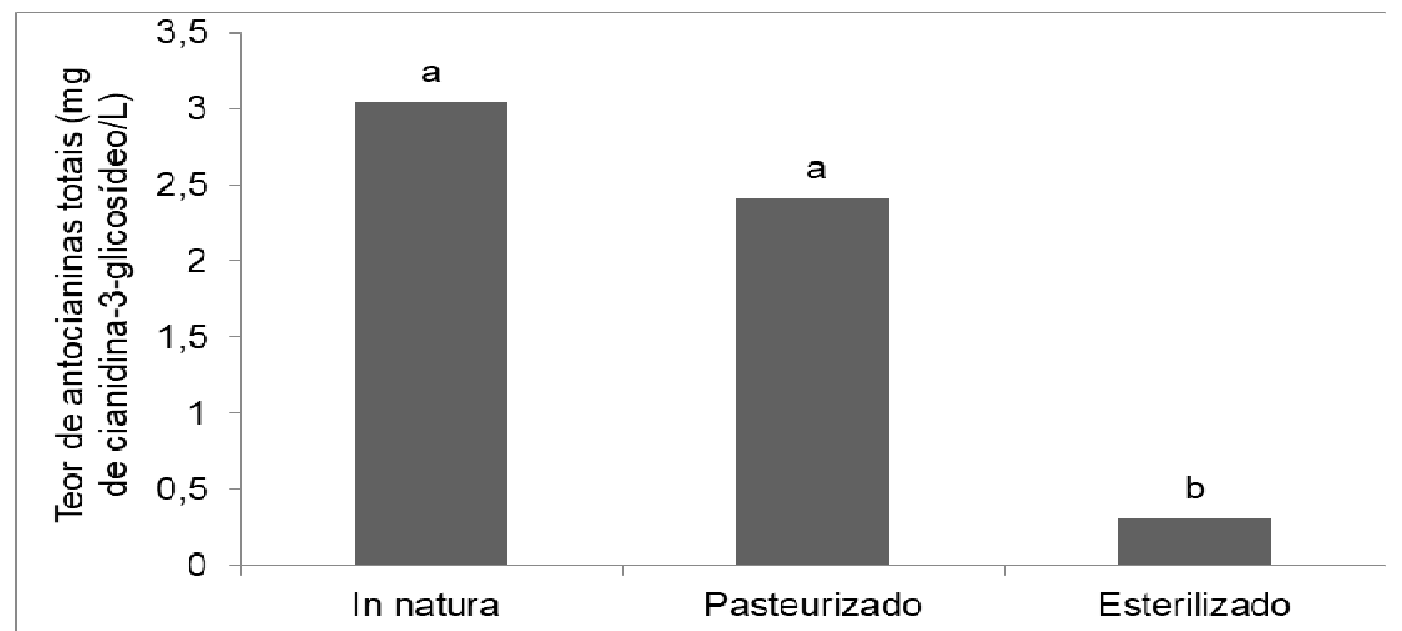

*Médias seguidas de mesma letra não diferem entre si, ao nível de 5\% de significância pelo teste de Tukey.

Figura 1: Médias do teor de antocianinas totais (mg/L), em bebidas de cálices de vinagreira. 
Considerando os valores absolutos para efeitos de cálculo, a retenção de antocianinas totais na bebida esterilizada foi de $10,19 \%$, quando comparado ao valor do produto in natura, corroborando assim, a influência do tratamento térmico na degradação de antocianinas, conforme descrito por Alvarenga et al. (2017), para blends de capuchinha e maracujá. O decréscimo no teor de antocianinas totais, em produtos processados, pode estar relacionado a fatores que envolvem altas temperaturas, variações de $\mathrm{pH}$ e concentrações de oxigênio, responsáveis por afetar a estabilidade deste componente bioativo, levando à degradação durante o processamento e consequentemente, proporcionando mudanças na coloração (CHAN, YAMAMOTO, 1994; MAIA et al., 2007).

\subsection{Análise Sensorial}

Em todos os atributos avaliados houve diferença significativa $(\mathrm{p}<0,05)$ para o tratamento esterilizado (Tabela 3), o que indica que o tratamento térmico influenciou a aceitação das bebidas de cálices de vinagreira.

Tabela 3: Médias de aceitação para a bebida de cálices de vinagreira in natura, pasteurizada e esterilizada.

\begin{tabular}{|c|c|c|c|c|}
\hline \multirow[b]{2}{*}{ Bebidas } & \multicolumn{4}{|c|}{ Parâmetros Sensoriais } \\
\hline & Cor & Sabor & $\begin{array}{c}\text { Impressão } \\
\text { Global }\end{array}$ & $\begin{array}{l}\text { Intenção de } \\
\text { Compra* }\end{array}$ \\
\hline In natura & $7,5^{\mathrm{a}}$ & $6,0^{\mathrm{a}}$ & $6,4^{\mathrm{a}}$ & $4,5^{\mathrm{a}}$ \\
\hline Pasteurizado & $7,4^{\mathrm{a}}$ & $6,0^{\mathrm{a}}$ & $6,4^{\mathrm{a}}$ & $4,3^{\mathrm{a}}$ \\
\hline Esterilizado & $4,9^{\mathrm{b}}$ & $4,1^{\mathrm{b}}$ & $4,4^{\mathrm{b}}$ & $2,0^{\mathrm{b}}$ \\
\hline
\end{tabular}

* Avaliado por meio de escala de 5 pontos, com os extremos "certamente compraria" e "certamente não compraria".

**Médias seguidas de mesma letra não diferem entre si, ao nível de 5\% de significância pelo teste de Tukey $(\mathrm{p}<0,05)$.

Para os atributos cor, sabor e impressão global, somente o tratamento esterilizado diferiu dos demais e obteve a menor média de aceitação, configurando rejeição dos avaliadores. As bebidas in natura e pasteurizada não diferiram significativamente entre si e foram estatisticamente, superiores, à bebida esterilizada. De acordo com Ferreira et al. (2005), a aplicação de calor suficiente para a destruição de microrganismos ou inativação das enzimas, podem acarretar alterações indesejáveis nos produtos, como por exemplo nas características sabor e coloração. Essas alterações podem ser associadas, provavelmente à degradação de compostos termo sensíveis, que conferem as qualidades nutricional e sensorial de frutas (PALLET et al. 2005).

Os resultados da intenção de compra foram condizentes com os da aceitação sensorial, descritos acima. A bebida que obteve menor escore foi a proveniente do tratamento esterilizado ("provavelmente não compraria"). Dessa forma, em todos os quesitos avaliados, os consumidores rejeitaram a bebida proveniente desse tratamento. Já para as bebidas in natura e pasteurizadas, observou-se que houve intenção de compra positiva, ficando os escores localizados entre "provavelmente compraria" e "certamente compraria".

Observou-se que as bebidas com melhor desempenho sensorial foram as que não receberam nenhum tratamento térmico (bebida in natura) e as pasteurizadas. Entretanto, considerando que, para fins comerciais, o prolongamento da vida útil de um produto é um fator econômico a ser ponderado, recomenda-se que para o suco elaborado, deve-se aplicar a pasteurização como alternativa viável ou avaliar o uso de outras técnicas de conservação, que não utilizam o calor. 


\section{CONCLUSÕES}

Pode-se concluir que não houve influência do tratamento térmico, da bebida formulada com cálices de vinagreira sobre a acidez total titulável, os sólidos solúveis totais e os sólidos totais.

A aplicação da esterilização como processamento térmico promoveu a degradação de antocianinas e a alteração da cor da bebida de vinagreira.

A pasteurização não promoveu alterações nas características físico-químicas e sensoriais, sendo similar à bebida de vinagreira que não foi submetida a nenhum tratamento térmico.

A comparação, entre os tratamentos térmicos usados, mostra que pasteurização é o mais indicado para a bebida de cálices de vinagreira, já que esta não diferiu da bebida in natura, em relação às características físico-químicas e sensoriais, apresentando maiores médias de aceitabilidade.

\section{REFERÊNCIAS}

ALVARENGA, G. F.; ALMEIDA-CARLOS, L.; ARRUDA, A.C.; MARTINS, L. M.; OLIVEIRA, K. G.; SILVA, E.C. Blend de maracujá e capuchinha: efeito do processamento térmico sobre compostos bioativos e características sensoriais. Brazilian Journal of Food Research, v. 8, p. 112 - 125, 2017.

AOAC - ASSOCIATION OF OFFICIAL ANALYTICAL CHEMISTRY. Official methods of analysis of the Association of Official Analytical Chemistry, Gaithersburg, Maryland. 3000p., 2012.

BRITO, A.C.W.; JUNIOR, B.D.; STERTZ, S.C.; FREITAS, R.J.S. Caracterização e estabilidade de purê misto de frutas, fonte de fibra alimentar. B. CEPPA v. 29, n 1, p. 137-146, 201. Disponível em: http://dx.doi.org/10.5380/cep.v28i1.22785 > acesso em 14/05/18

CARDOSO, L.M.; LEITE, J.P.V.; PELUZIO, M.C.G. Efeitos biológicos das antocianinas no processo aterosclerótico. Revista Colombiana de Ciência Químico-Farmacêutica. v. 40, n.1, p. 116-138, 2011.

CARVAlHO, P.G.B.; MACHADO, C.M.M.; MORETTI, C.L.; FONSECA, M.E.N. Hortaliças como alimentos funcionais. Horticultura Brasileira, v. 24, n.4, p. 397-404, 2006.

CHAN, H. T.; YAMAMOTO, H. Y. Kinetics of anthocyanin decomposition in acerola juice. Asean Food J., v. 9, n. 4, p. 132-135, 1994.

CIANCI, F.C.; SILVA, L.F.M.; CABRAL, L.M.C.; MATTA, V.M. Clarificação e concentração de suco de caju por processos com membranas. Ciência e Tecnologia de Alimentos, v. 25, n.3, p. 579-583, 2005.

DAMASCENO, L.F.; FERNANDES, F.A.N.; MAGALHÃES, M.M.A.; BRITO, E.S. Non-enzymatic browning in clarified cashew apple juice during Thermal treatment: Kinetics and process control. Food Chemistry, v. 106, p.172-179, 2008.

FERREIRA, L. S.; JARDIM, F. B. B.; MIGUEL, D. P. Estudo do efeito da esterilização comercial na qualidade microbiológica e sensorial de Corned beef. Fazu, n. 2, p. 136-143, 2005.

FIORE, A.; FAUCI, L.L.; CERVELLATI, R.; GUERRA, M.C.; SPERONI, E.; COSTA, S.; GALVANO, G.; LORENZO, A.; BACHELLI, V.; FOGLIANO, V.; GALVANO, F. Antioxidant activity of pasteurized and sterilized commercial red orange juices. Molecular Nutrition \& Food Research, v. 49, p. 1129-1135, 2005. Disponível em https://onlinelibrary.wiley.com/doi/epdf/10.1002/mnfr.200500139. 
FONSECA, A. C. M.; SEDIYAMA, M. A. N.; FONTANÉTTI, A.; PEDROSA, M. W.; SANTOS, I.C. Azedinha, capuchinha e vinagreira: cores e sabores que alimentam. INFORME AGROPECUÁRIO (BELO HORIZONTE). v.37, p. $53-66,2016$.

GIUSTI, M. M.; WROLSTAD, R. E. Anthocyanins. Characterization and Measurement with UV-Visible Spectroscopy. In: Wrolstad, R. E. (Ed.). Current Protocols in Food Analytical Chemistry. New York: John Wiley \& Sons, Unit. F1.2.p. 1-13, 2001.

GOBBI, K. R.; ALMEIDA, L.R.; SCHIRMER, M.; FARIA, R. A. P. G. Qualidade físico-química de néctares industrializados comercializados em Cuiabá--MT. Brazilian Journal of Food Research, v. 7, p. 1628, 2016.

JIAO, B.; CASSANO, A.; DRIOLI. E. Recent advances on membrane processes for the concentration of fruit juices: a review. Journal of Food Science, v. 63, p. 303-324, 2004.

KINUPP, V.F.; BARROS, I.B.I. Teores de proteína e minerais de espécies nativas, potenciais hortaliças e frutas. Ciência e Tecnologia de Alimentos, Campinas, v. 28, n.4, p. 846-857, 2008.

KINUPP, V. F.; LORENZI, H. Plantas alimentícias não convencionais (PANC) no Brasil: guia de identificação, aspectos nutricionais e receitas ilustradas. Nova Odessa: Instituto Plantarum de Estudos da Flora. 768p. 2014.

MAIA, G.A.; SOUSA, P.H.M.; SANTOS, GM.; SILVA, D.S.; FERNANDES, A.G.; PRADO, G.M. Efeito do processamento sobre componentes do suco de acerola. Ciência e Tecnologia de Alimentos, v. 27, n.1, p. 130-134, 2007.

MINISTÉRIO DA AGRICULTURA PECUÁRIA E ABASTECIMENTO (MAPA). Manual de hortaliças não-convencionais. 1. ed. Brasília: MAPA/ACS, 95 p., 2010.

NACHTIGALL, A.M.; ZAMBIAZI, R.C. Geleias de hibisco com reduzido valor calórico: Características sensoriais. Centro de Pesquisa e Processamento de Alimentos. v. 24, n. 1, p. 47-58, 2006.

OLIVEIRA, D.C.S.; WOBETO, C.; ZANUZO, M.R.; SEVERGNINI, C. Composição mineral e teor de ácido ascórbico nas folhas de quatro espécies olerícolas não-convencionais. Horticultura Brasileira, v. 31, n. 3, p. 472-475, 2013.

PALlET, D.; CABRAL, L.; MATTA, V.; PEZOA-GARCÍA, N.H.; MENEZES, H.C.; ABREU, F.A.P.; DORNIER, M.; REYNES, M. Aplicação de tecnologia de membranas no processamento de sucos de frutas brasileiras. Caderno de Ciência \& Tecnologia, v. 22, n. 2, p. 427-437, 2005.

REIS, R.C.; MINIM, V.P.R.; Teste de aceitação. In: MINIM, V.P.R. (Ed.). Análise Sensorial: Estudos com consumidores. Viçosa: Editora UFV, Cap. 3, p.65-81, 2013.

SILVA, M.L.C.; COSTA, R.S.; SANTANA, A.S.; KOBLITZ, M.G.B.; Compostos fenólicos, carotenoides e atividade antioxidante em produtos vegetais. Semina: Ciências Agrárias, v. 31, n.3, p. 669-682, 2010.

VERA, E.; RUALES, J.; DORNIER, M.; SANDEAUX, J.; PERSIN, F., PORCELLY, G.; VAILLANTE, F.; REYNES, M. Comparison of different methods for deacidification of clarifield passion fruit juice. Journal of Food Engineering. v. 59, p. 361-367, 2003.

VILLADIEGO, A.M.D. Fundamentos de estabilidade de alimentos. 2. ed. Brasília, Embrapa, 326 p. 2012. 
VIZZOTTO, M.; PEREIRA, M.C. Hibisco: do uso ornamental ao medicinal. 2008. Artigo em Hypertexto. Disponível em: <http://www.infobibos.com/Artigos/2008_4/hibisco/index.htm>. Acesso em: 02/05/2018.

WANG, C.J.; WANG, J.M.; LIN, W.L.; CHU, C.Y.; CHOU, F.P.; TSENG, T.H. Protective effect of hibiscus anthocyanins againt tert-butyl hydroperoxide-induced hepact toxicity in rats. Food and Chemical Toxicology. v. 38, n.5, p. 411-416, 2000. 